\title{
CARACTERÍSTICAS PERSONALES DEL MIGRANTE Y EL CONSUMO DE DROGAS LICITAS E ILÍCITAS: UNA REVISIÓN SISTEMÁTICA
}

PERSONAL CHARACTERISTICS OF THE MIGRANT AND THE USE OF LEGAL AND ILLEGAL DRUGS: A SYSTEMATIC REVIEW

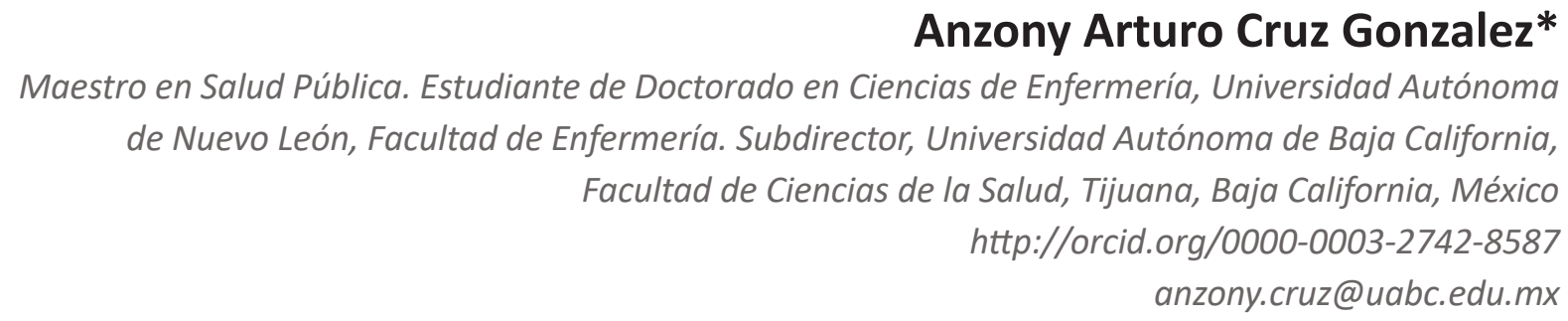

María Magdalena Alonso Castillo**

Doctora en Filosofía con especialidad en Psicología. Profesora de tiempo completo y exclusivo, Universidad Autónoma de Nuevo León, Facultad de Enfermería, Monterrey, Nuevo León, México https://orcid.org/0000-0002-7197-8116 magdalena_alonso@hotmail.com

\section{Nora Angélica Armendáriz García}

Doctora en Ciencias de Enfermería. Profesora de tiempo completo, Universidad Autónoma de Nuevo León, Facultad de Enfermería, Monterrey, Nuevo León, México https://orcid.org/0000-0001-9033-3244 nordariz@hotmail.com

Francisco Rafael Guzmán Facundo

Doctor en Enfermería. Profesor de tiempo completo. Universidad Autónoma de Nuevo León, Facultad de Enfermería. Monterrey, Nuevo León, México https://orcid.org/0000-0002-6951-8989 pako2001@hotmail.com

*Autor principal ${ }^{* *}$ Autor principal correspondencia

Cómo citar este texto:

Cruz Gonzalez, A.A., Alonso Castillo, M.M., Armendáriz García, N.A. y Guzmán Facundo, F.R. (2022).

Características personales del migrante y el consumo de drogas licitas e ilícitas: una revisión sistemática. Health and Addictions / Salud y Drogas, 22(1), 95-107. doi: 10.21134/haaj.v22i1.619 


\title{
Resumen
}

Introducción. La migración tiene efecto en los ámbitos individuales, sociales y familiares, estos efectos requerirán de procesos de adaptación que afectan los estilos de vida, se verán reflejados en los procesos de salud, enfermedad y en conductas de riesgo como el consumo de drogas. Objetivo. Determinar las características personales del migrante que pueden influir en el consumo de drogas licitas e ilícitas. Metodología. Se siguieron los pasos del Manual Crochrane y los Elementos de Informes para la Presentación de Revisiones Sistemáticas, como estrategia de búsqueda, se incluyeron los descriptores Desc el tesauro Mesh en inglés y español, así como truncadores y operadores boléanos en el título y resumen: Migrants AND Alcohol Drinking OR Drugs Abuse. Resultados. Los 25 estudios que integran la revisión sistemática comprenden características personales del migrante como edad, genero, escolaridad, ocupación, estado civil asociadas al consumo de drogas, de los cuales el $72 \%$ consideran características personales asociadas al consumo de alcohol, $12 \%$ al tabaco, $8 \%$ a drogas ilícitas y $8 \%$ a distintos tipos de drogas. Conclusiones. Los resultados de esta revisión sistemática aportan evidencia entre la relación de algunas características personales de los migrantes como factores de riesgo que influyen el consumo de drogas licitas e ilícitas.

\begin{abstract}
Introduction. Migration has an effect on the individual, social and family spheres, these effects will require adaptation processes that affect lifestyles, they will be reflected in the processes of health, disease and risk behaviors such as drug use. Objective. Determine the personal characteristics of the migrant that can influence the consumption of legal and illegal drugs. Methodology. The steps of the Crochrane Manual and the Elements of Reports for the Presentation of Systematic Reviews were followed, as a search strategy, the descriptors Desc the Mesh thesaurus in English and Spanish were included, as well as truncators and Boléan operators in the title and abstract: Migrants AND Alcohol Drinking OR Drugs Abuse. Results. The 25 studies that make up the systematic review include personal characteristics of the migrant such as age, gender, education, occupation, marital status associated with drug use, of which $72 \%$ consider personal characteristics associated with alcohol consumption, $12 \%$ with tobacco. $8 \%$ to illicit drugs and $8 \%$ to different types of drugs. Conclusions. The results of this systematic review provide evidence between the relationship of some personal characteristics of migrants as risk factors that influence the consumption of licit and illicit drugs.
\end{abstract}

\section{Palabras clave}

Alcoholismo; drogas ilícitas; migrantes; Tabaco.

\section{Keywords}

Alcoholism; illicit drugs; migrants; Tobacco. 


\section{INTRODUCCIÓN}

La migración es un fenómeno con tradición histórica, que requiere de nuevas aristas para su explicación, en virtud de las múltiples razones para emigrar como el deseo de prosperidad, la búsqueda de mejores condiciones de vida, de poder solventar las necesidades de la familia, además, de motivos de seguridad y para el reencuentro con sus seres queridos que ya han migrado al país de destino, no obstante en este proceso se enfrentaran a diversas situaciones y hechos que pueden afectar su bienestar (Simkin, 2020; Wickramage et al., 2018).

El Instituto Nacional de Estadística y Geografía (INEGI, 2014) calcula que la proporción de la población migrante internacional según su lugar de destino, hacia los Estados Unidos de América es del 89.4\%, a otros países $7 \%$ y no determinado 3.6\%. La migración tiene un efecto en los ámbitos individuales, sociales y familiares, estos efectos o consecuencias requerirán de procesos de adaptación que afectan los estilos de vida de la persona migrante y su familia, del mismo modo se verán reflejados estos efectos en los procesos de salud y enfermedad y en las conductas de riesgo como el consumo de drogas; además de trascender las áreas de atención y servicios de salud, por causa del consumo de drogas licitas e ilícitas (Piñones-Rivera, Concha \& Gómez, 2021).

El consumo de drogas es un fenómeno que se incrementa año con año, se estima que en el 2018, de la población mundial con edades comprendidas entre 15 a 64 años, 269 millones de personas (5.3\%) consumen algún tipo droga; referente al consumo de drogas ilícitas a nivel mundial, 192 millones de personas consumieron cannabis en el 2018, por lo que es la droga más consumida, sin embargo, 58 millones de personas consumieron opioides causando el $66 \%$ de las muertes relacionadas con el consumo de drogas ilícitas, por lo que los opioides son la droga ilícita más nociva (World Drug Report, 2020).

A nivel mundial, el consumo de alcohol es causa del 5.1\% de las enfermedades y responsable de 3.3 millones de muertes cada año, además, de ser causantes de las repercusiones físicas, psicológicas y sociales provocadas por este consumo. En cuanto al consumo de tabaco, aunque el número de consumidores a nivel mundial ha disminuido, se reportan en 2018, un total de 1337000 millones de consumidores de tabaco, 8 millones de muertes a causa del consumo, de las cuales, 7 millones de muertes son producto del consumo directo (Organización Mundial de Salud [OMS], 2018).

Actualmente se considera que existen factores de riesgo en el proceso migratorio que pueden influir en el fenómeno del consumo de drogas licitas e ilícitas, su importancia radica, en el aumento de la probabilidad de exposición al fenómeno de la drogodependencia, considerado un problema de salud pública, por los daños a la salud física, psicológica y social, por su magnitud cada vez más incremental y que demanda de servicios de salud muchas veces no disponibles para este grupo. Estos factores de riesgo se definen como características intrínsecas y extrínsecas, condiciones y contextos del migrante, que aumenta la probabilidad de iniciar en el uso y/o abuso de drogas, así como en la modificación del patrón de consumo. Dentro de algunos factores de riesgo para consumo de drogas en la población migrante se encuentran los siguientes: las características personales del migrante como edad, género, estado civil, ocupación y escolaridad que podrían incrementar la vulnerabilidad al consumo de drogas, así como, el estrés migratorio, el temor a ser deportado, la falta redes sociales de apoyo durante proceso migratorio, el miedo a no poder llegar a su lugar de destino, la falta de recursos para subsistir mientras realiza este proceso, el maltrato de diferentes personas y el temor a las autoridades por su condición migratoria (Becoña Iglesias et al., 2002; Calafat et al., 2005; Vázquez \& Becoña, 2000).

Se ha identificado relación positiva y significativa entre algunas características personales del migrante y el consumo de drogas licitas e ilícitas, el género se ha descrito como un factor asociado al consumo de drogas en esta población, reportando mayor consumo en el caso de los hombres en comparación con las mujeres migrantes (Lopez-Tamayo et al., 2016; Sánchez-Huesca \& Arellanez-Hernández, 2011; Tortajada Navarro et al., 2008).

Respecto a los patrones de consumo se reportó que los factores que actuaron como riesgo para el consumo de alcohol fueron: el estatus laboral antes de migrar, el lugar donde espera antes de llegar al sitio de destino y la condición irregular en el lugar del destino final (Sánchez-Huesca et al., 2006). Se documentó que otros factores como tener 
una pareja migrante que consume alcohol, incrementa cuatro veces la probabilidad de consumo versus no tener conyugues consumidores. En relación con el consumo excesivo de alcohol en el año previo a la migración en mujeres migrantes el haberse separado de su pareja durante el proceso migratorio y tener un nivel educativo básico de primaria o menos fueron factores de riesgo (Liu et al., 2017). En algunos estudios se ha descrito al estrés migratorio que experimentan los migrantes en este proceso el que se relaciona positiva y significativamente con el consumo de drogas (Arellanez-Hernández, 2016; Muñoz-García \& Arellanez-Hernández, 2015; Zapata, Grzywacz, Cervantes \& Merten, 2016).

Es fundamental analizar la literatura existente debido a la falta de información sintetizada y con rigor metodológico en su búsqueda, para que pueda hacerse uso de ella, ya que se ha identificado diversificación en la evidencia e inconsistencias en los resultados de algunas investigaciones, por ejemplo pertenecer al género masculino se identificó en la mayoría de los artículos (De La Rosa et al., 2020; Levitt et al., 2019; Cano et al., 2018; Walsh et al., 2018; Blackson et al., 2015; Bryant \& Kim, 2013; Dillon et al., 2013), sin embargo, de forma contraria, algunos artículos reportan al género femenino como un factor de riesgo del consumo de alcohol (Blackson et al., 2015; Sánchez, 2015; Park et al., 2014), así mismo existen contradicciones sobre factores como escolaridad, estado civil e ingreso económico, entre otros (Di Napoli et al., 2020; Liu et al., 2017; Sordo et al., 2015). Por lo anterior, es importante realizar una identificación, evaluación y síntesis de la evidencia basada en la investigación y reportarla en un formato accesible (Mulrow 1994), de tal manera que se indiquen las características personales de los migrantes que pueden influir en el consumo de drogas licitas e ilícitas. El producto de este análisis podrá ser de utilidad para el personal de salud y en un futuro desarrollar e implementar programas específicos de prevención, promoción de la salud y el fortalecimiento de aquellos factores que permitan el control de los riesgos. Por lo señalado anteriormente, el objetivo de este estudio es determinar las características personales del migrante que pueden influir en el consumo de drogas licitas e ilícitas.

\section{MÉTODO}

Para la realizar la presente revisión sistemática se siguieron las recomendaciones según los pasos propuestos por el Manual Crochrane y los Elementos de Informes para la Presentación de Revisiones Sistemáticas (PRISMA, por sus siglas en inglés). Como estrategia de búsqueda se incluyeron los descriptores Desc el tesauro Mesh en los idiomas inglés y español en todas las combinaciones posibles, así como truncadores y operadores. El resultado total de la estrategia de búsqueda se registra en la Tabla 1. La selección de artículos se ejecutó en dos momentos, primero se revisó el título y el resumen de cada uno de los artículos localizados y un segundo momento se llevó a cabo la lectura del texto completo. Se utilizó la biblioteca Zotero para la concentración de referencias completas.

Dentro de los criterios de elegibilidad se incluyeron todos aquellos estudios que cumplieron con las siguientes características:

Diseño; se incluyeron estudios con metodología de tipo descriptivo, correlacional, predictivo y de diseño mixto que fueron publicados sin restricción de tiempo en su publicación y que tuvieran como objetivo evaluar el consumo de drogas en la población migrante como alcohol, tabaco, y drogas ilícitas como marihuana, cocaína, drogas de síntesis entre otros, sin embargo, solo se reportaron los resultados con respecto al alcohol, tabaco, metanfetaminas, cocaína y cannabis, y sus posibles factores de riesgo.

Participantes; todos aquellos estudios que incluyeron población migrante dentro de diferentes grupos que migran en Estados Unidos de América (14), China (4), España (2), Países Bajos (1), Israel (1), Italia (1), Suecia (1) y Canadá (1), como parte de los estudios analizados en la presente revisión.

Idioma; la búsqueda de los artículos se llevó a cabo en los idiomas inglés y español, en la Tabla 1 se muestra algunos ejemplos de los términos utilizados y los operadores boléanos empleados como otra herramienta de búsqueda. 


\section{Estrategia de búsqueda}

Se desarrolló una estrategia de búsqueda electrónica comprensiva y claramente estructurada para obtener la mejor evidencia en las diversas bases de datos. La búsqueda se llevó a cabo durante los meses de abril a Julio de 2020; se consultaron las siguientes bases de datos: EBSCO, PubMed, Scielo, Scopus y LiLACS, además de estudios adicionales publicados en Google académico. Como estrategia de búsqueda se incluyeron los descriptores Desc y el tesauro Mesh en los idiomas inglés y español en todas las combinaciones posibles, así como truncadores y operadores boléanos en el título y el resumen: Migrants AND Alcohol Drinking OR Drugs Abuse. Se utilizó la biblioteca Zotero para la concentración de referencias. Al realizar las búsquedas se identificaron artículos entre las bases de datos, posteriormente se procedió a leer los títulos y resúmenes para seleccionar los artículos para ser analizados.

Tabla 1. Resultados de la estrategia de búsqueda.

\begin{tabular}{|c|c|c|}
\hline Base de datos & Descriptores Desc y el tesauro Mesh & Número de resultados \\
\hline EBSCO host & Immigrant AND beer OR drug use disorder OR Tobacco & 263 \\
\hline PubMed & Migrant AND alcohol abuse OR designer drugs OR Tobacco & 77 \\
\hline Scielo & Migrant worker AND alcohol addiction OR drug addiction OR Tobacco & 2 \\
\hline Scopus & Transients and Migrants AND alcohol dependence OR Drug Dependence OR Tobacco & 104 \\
\hline LiLACS & Emigrant AND alcoholism OR Drug Habituation OR Tobacco & 98 \\
\hline Google académico & Migrants AND Alcohol Drinking OR Drugs Abuse OR Tobacco & 2 \\
\hline
\end{tabular}

\section{Proceso de recolección}

Una vez probada la estrategia de búsqueda, se procedió a replicarla en las diversas bases de datos; se obtuvieron 544 artículos mediante esta fuente, se incluyeron adicionalmente 2 artículos obtenidos a través de la búsqueda en literatura gris lo que dio como resultado un total 546 artículos. Se utilizó un administrador bibliográfico para organizar las referencias localizadas y poder eliminar los duplicados, obteniendo un registro de 222 artículos. Como primer paso, se realizó un screenining o cribado de los datos por título dando un resultado de 143 artículos y por resumen 70 artículos, posteriormente, se ubicaron aquellos artículos con información relevante y se procedió a leerlos a texto completo e incluir los que cumplieran con los criterios de elegibilidad en la muestra final. En cada etapa del proceso se especifican las razones por las cuales algunos artículos fueron eliminados. Lo anterior se realizó con base a lo especificado en el diagrama de flujo del PRISMA (figura 1). 
Figura 1. Diagrama de flujo PRISMA modificado (Liberati et al., 2009).
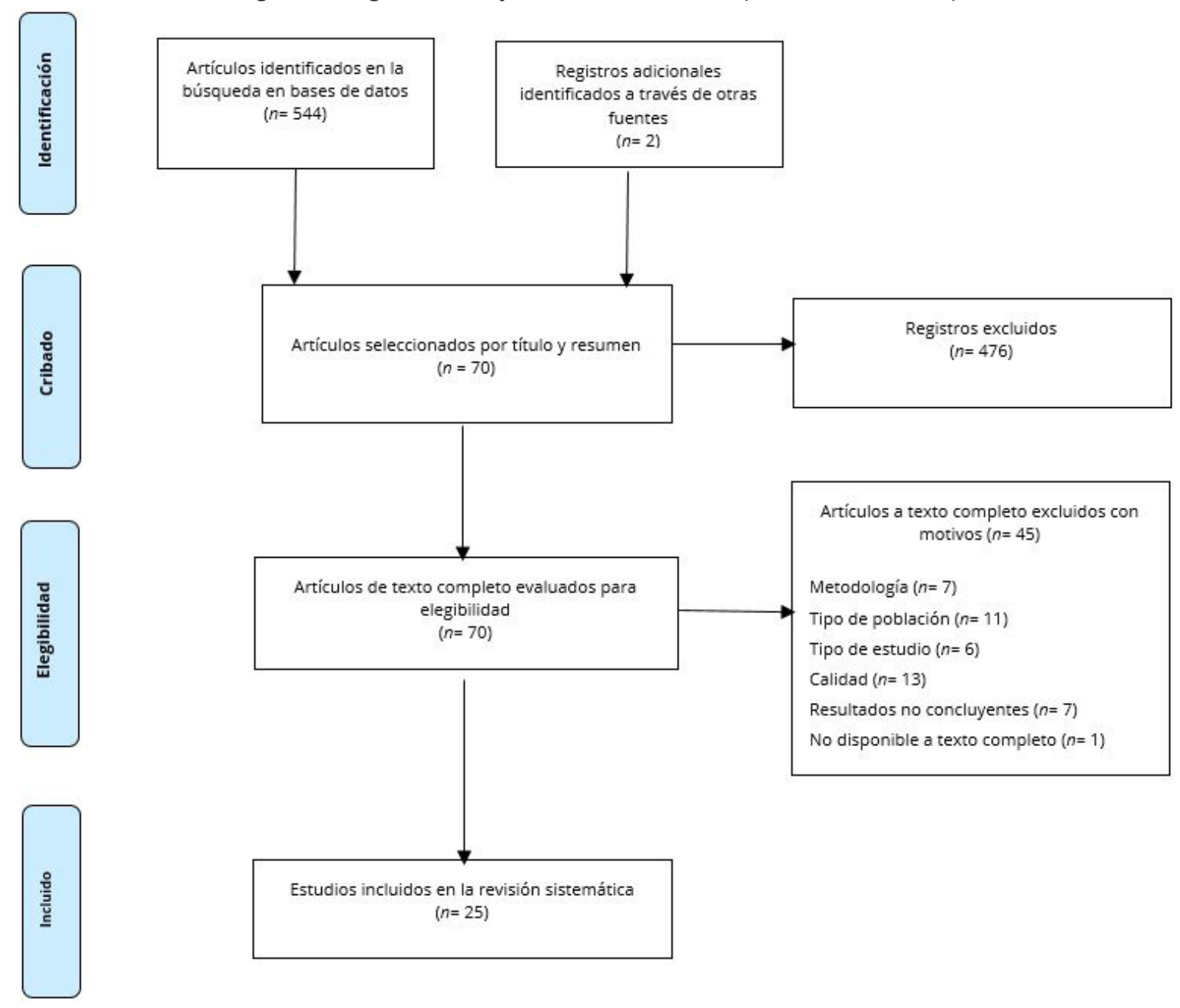

\section{Extracción de datos}

Se utilizó STROBE (Strengthening the Reporting of Observational studies in Epidemiology), con la finalidad de obtener información relevante de los artículos incluidos, que permite evaluar la calidad de los artículos, así como integrar de manera esquematizada la información relevante de cada estudio evaluado.

\section{RESULTADOS}

Se destaca que los estudios utilizan una amplia gama de instrumentos para la recolección de datos, y existe diversificación en los resultados según los distintos autores. La totalidad de los estudios localizados fue de 546 artículos, de los cuales se incluyen en esta revisión sistemática 25 en los que se identificó asociación entre características personales del migrante y el consumo de drogas licitas e ilícitas. Los estudios que integran la revisión sistemática comprenden características personales del migrante como edad, género, escolaridad, ocupación y estado civil que se asocian al consumo de drogas licitas e ilícitas en esta población, de los cuales el $72 \%$ consideran características personales asociadas al consumo de alcohol, el $12 \%$ al consumo de tabaco, el $8 \%$ al consumo de drogas ilícitas (metanfetaminas, cocaína y cannabis) y el $8 \%$ analiza distintos tipos de consumo de drogas en conjunto (alcohol, tabaco, cannabis y cocaína).

Los estudios encontrados se realizaron en mayor proporción en Estados Unidos de América con un 56\% (Arcury et 
al., 2016; Blackson et al., 2015; Bryant \& Kim, 2013; Cano, 2020; Cano et al., 2018; Cantrell, 2014; De La Rosa et al., 2020; Dillon et al., 2013; Hernández et al., 2009; Levitt et al., 2019; Marsiglia et al., 2009; Park et al., 2014; Patel et al., 2018; Sánchez, 2015), seguidos de China con un 16\% (Chen \& Chien, 2018; Liu et al., 2015; Liu et al., 2017; Zaller et al., 2014), España con un 8\% (Sordo et al., 2015; Tortajada Navarro et al., 2008), mientras que en menor proporción en Países Bajos (Creemers et al., 2017), Israel (Walsh et al., 2018), Italia (Di Napoli et al., 2020), Suecia (Harris et al., 2019) y Canadá (Hamilton et al., 2012) con un $4 \%$ cada uno, correspondiendo a estudios de tipo no experimental. La población está integrada sin restricciones de edad de 11 años o más, constituida por migrantes hispanos, trabajadores migrantes latinos, jóvenes migrantes con diversos orígenes étnicos (Surinam, Marroquís, Turco, Antillano y Asiático), migrantes indios, migrantes asiáticos, migrantes en España y migrantes no refugiados, en estudios existentes del año 2008 al 2020, de los cuales $48 \%$ fueron publicados en los último cinco años, en el periodo comprendido de $2016-2020$, el $40 \%$ publicados entre el $2011-2015$ y $12 \%$ publicados en el periodo del 2008-2010.

\section{Consumo de alcohol}

Referente a los artículos que se centran en el consumo de alcohol, en relación con la edad, tres artículos documentaron que el tener edades comprendidas entre 11 a 60 años se relaciona con el consumo de alcohol (Walsh et al., 2018; Sordo et al., 2015; Zaller et al., 2014; Bryant \& Kim, 2013). El pertenecer al género masculino se identificó en 9 de los artículos (De La Rosa et al., 2020; Levitt et al., 2019; Cano et al., 2018; Walsh et al., 2018; Blackson et al., 2015; Bryant \& Kim, 2013; Dillon et al., 2013), sin embargo, de forma contraria, 3 de los artículos analizados reportan al género femenino como un factor de riesgo asociado con el consumo de alcohol (Blackson et al., 2015; Sánchez, 2015; Park et al., 2014). Respecto a la escolaridad, existe disparidad en los resultados, ya que se reporta que los niveles educativos bajos se asocian con el consumo de alcohol (Liu et al., 2017; Dillon et al., 2013), mientras en otro artículo reconocen a los niveles altos como factor de riesgo para el consumo de alcohol (Bryant \& Kim, 2013).

De igual forma, referente al estado civil, existe discrepancia en los resultados, ya que estudios reportan que el estar casado, tener pareja y/o hijos, y el tener cónyuges que consumen alcohol es un factor que se asoció al consumo de alcohol (Liu et al., 2017; Bryant \& Kim, 2013; Sordo et al., 2015), mientras de forma contraria otros artículos documentan el ser separado, divorciado, viudo o soltero como un factor de riesgo vinculado al consumo de alcohol (Di Napoli et al., 2020; Liu et al., 2017). Respecto a los factores familiares, existe diferencias en lo citado entre las referencias, ya que unos artículos declaran la permisividad de los padres o un mayor ingreso familiar (De La Rosa et al., 2020; Cano et al., 2018; Creemers et al., 2017), sin embargo, de forma contraria otros artículos documentan a los conflictos familiares se asociaron con una mayor frecuencia de consumo de alcohol (Park et al., 2014; Marsiglia et al., 2009).

Asimismo, el uso de otras drogas se relacionó con el uso de alcohol (Walsh et al., 2018; Liu et al., 2017; Sordo et al., 2015), de igual forma, tener compañeros que consumen alcohol se relaciona con el consumo de alcohol (Creemers et al., 2017). Además, referente al estado de salud, percibir un estado de salud regular, pobre o muy pobre, el estrés migratorio, estar moderada o severamente deprimida, el tener síntomas depresivos, los trastornos del estado de ánimo son factores de riesgo que se asocian al consumo de alcohol (Di Napoli et al., 2020; Harris et al., 2019; Arcury et al., 2016; Sánchez, 2015; Sordo et al., 2015; Zaller et al., 2014; Bryant \& Kim, 2013).

Respecto al estatus laboral, ser trabajador agrícola, trabajar en un lugar de nivel socioeconómico alto y estar desempleado son factores de riesgo que se asocian al consumo de alcohol (Di Napoli et al., 2020; Liu et al., 2017; Arcury et al., 2016; Sordo et al., 2015; Zaller et al., 2014). Además, aspectos culturales durante la migración o la inserción cultural, como la cultura étnica, la aculturación, la enculturación, la discriminación étnica percibida, tener un mayor dominio del idioma inglés, estatus migratorio, ser de origen Mexicano, vivir en un campamento de migrantes, el tiempo en Estados Unidos, vivir en el centro de España, vivir en zonas urbanas con más de 50000 habitantes, la duración de la estadía > 7 años se asociaron significativamente con los patrones de consumo de alcohol (Cano, 2020; Di Napoli et al., 2020; Chen \& Chien, 2018; Walsh et al., 2018; Sánchez, 2015; Sordo et al., 2015; Park et al., 2014; Bryant \& Kim, 2013; Dillon et al., 2013; Marsiglia et al., 2009).

Finalmente, el divertirse, la inseguridad, ser religioso o muy religioso, el haber tenido alguna exposición a delincuen- 
cia, violencia o vandalismo o una mejor situación financiera son algunos motivos para consumo de alcohol (Sánchez, 2015; Sordo et al., 2015; Bryant \& Kim, 2013; Tortajada Navarro et al., 2008).

\section{Consumo de tabaco}

En cuanto a los artículos que analizan el consumo de tabaco, la diferencia más importante es la relacionada con el número de años viviendo en los Estados Unidos, ya que un artículo reporta el haber habitado 12 años en este país como un factor de riesgo para el consumo de tabaco (Patel et al., 2018), mientras que en otro artículo refiere que a partir de cuatro años como factor de riesgo relacionado al consumo de tabaco (Liu et al., 2015). En el caso de 2 artículos no hacen diferencias entre el género masculino y femenino, por lo anterior, el deterioro medio y alto de la salud mental, la educación en alguna universidad/escuela técnica, la educación de posgrado, el divertirse y por inseguridad son factores de riesgo para el uso de tabaco (Patel et al., 2018; Tortajada Navarro et al., 2008).

Mientras que dos autores hacen distinción entre características de la migración asociado con el consumo de tabaco, diferenciando entre hombres y mujeres; en el caso de las hombres: tener más de 35 años, percibir ingresos $\geq$ US\$40,000/año, trabajar en la construcción, el trabajar en el servicio doméstico, el trabajo en el sector del entretenimiento, ser divorciados/viudos, con una duración de la migración de 4 a 5 años, con una duración de la migración de más de cinco años, el número de tres ciudades migratorias, el número de cuatro ciudades migratorias y el número de $\geqq 5$ ciudades migratorias esta significativamente asociado con el consumo de tabaco (Liu et al., 2015; Cantrell, 2014).

En el caso de las mujeres: tener niños menores de 18 años, el trabajo en la construcción, trabajo en hoteles/restaurantes, el trabajo en el sector del entretenimiento, con ingresos mensuales $>3500$ yuanes y el número de dos ciudades migratorias está asociado significativamente con el consumo de tabaco (Liu et al., 2015; Cantrell, 2014).

\section{Consumo de drogas ilícitas (cannabis, cocaína, metanfetaminas)}

En relación con la edad, tres artículos documentan el tener edades comprendidas entre 12 a 34 años como factor de riesgo para el consumo de cannabis, cocaína y metanfetaminas (Walsh et al., 2018; Hernández et al., 2009; Hamilton et al., 2012). El tener de 2 a 4 parejas sexuales, tener más de 5 parejas sexuales, consumo de alcohol de 2 o más días, tener síntomas depresivos, cualquier Infección de Trasmisión Sexual y tener de media a alta aculturación se asoció significativamente con el consumo de metanfetamina y cocaína (Hernández et al., 2009); mientras que el divertirse y por inseguridad son los principales motivos para consumo de cannabis y cocaína (Tortajada Navarro et al., 2008). Además, la discriminación, que los padres no cuenten con un título universitario, el faltar a la escuela, el tener amigos que usan drogas se asoció con consumo de cannabis (Walsh et al., 2018; Hamilton et al., 2012).

\section{DISCUSIÓN}

Dentro de los aspectos que deben resaltarse, es que los resultados muestran que existe relación entre las características personales del migrante que actúan como factores de asociación o determinantes del consumo de drogas licitas e ilícitas; además existen diferencias entre la población de estudio en relación con la multiculturalidad (Kuo, 2014), en general se pueden destacar tres aspectos generales:

En primer lugar, la relación entre género y consumo de drogas, aunque la mayor parte de los estudios analizados declaran la relación entre el género masculino como factor de riesgo asociado con el consumo de drogas licitas e ilícitas, algunos artículos confirman un aumento en la relación de género femenino con el consumo de alcohol específicamente, con base a lo anterior, algunos autores identifican la trasformación del rol social femenino y los cambios en las normas sociales femeninas, con las que se adoptan comportamientos de riesgo para la salud (Kaya et al., 2016; Patró-Hernández et al., 2019), un problema que se identifica, es el uso de instrumentos sin diferenciar las características tanto para hombres como para mujeres, por ejemplo, las diferencias del consumo de alcohol 
entre hombres y mujeres es debido a que las mujeres alcanzan niveles de intoxicación de forma más rápida que el hombre con dosis equiparables, debido a que las mujeres cuentan con $14 \%$ menos agua que los hombres, por tanto el etanol al ser hidrosoluble se distribuye de forma uniforme; además, en el caso de los hombres, no se toma en cuenta aspectos referentes a la masculinidad, la dominancia, el donjuanismo, la búsqueda de admiración y posición social; en el caso de las mujeres, no se consideraron características como el interés por la crianza de los hijos, la fidelidad sexual, la dedicación al hogar o el romanticismo en las relaciones; estas diferencias requiere que dicha variable sea analizada con mayor detenimiento, poniendo énfasis en los instrumentos de medición adaptados para ambos géneros (Patró-Hernández et al., 2019).

En segundo lugar, escolaridad de los participantes, aunque la mayor parte de los estudios reportan a los niveles educativos bajos asociados con el consumo de alcohol (Dillon et al., 2013; Liu et al., 2017), en esta variable no se toma en cuenta el acceso a la educación de las personas migrantes especialmente en su condición de ilegalidad, por lo anterior se debe incluir en los instrumentos de medición una pregunta en la cédula de datos sociodemográficos que permita la identificación del estatus migratorio en los territorios donde se encuentran, ya que algunos artículos reconocen a los niveles altos como factor de riesgo para el consumo de drogas alcohol, sin indagar la accesibilidad de estos a continuar con sus estudios (Bryant \& Kim, 2013).

Por último, los factores familiares, se identifican en esta revisión, sobre todo aspectos referentes a la permisividad, cohesión, ingresos y los conflictos familiares (Cano et al., 2018; Creemers et al., 2017; De La Rosa et al., 2020; Marsiglia et al., 2009; Park et al., 2014), sin embargo, no se declara la estructura familiar y si la población en estudio son migrantes de primera, segunda o tercera generación, esta diferencia genera cambios en la dinámica y el núcleo familiar, además de lo anterior, los migrantes que viajan solos evidentemente no tienen exposición a este tipo de problemáticas por lo que es necesario diferenciarlos e indagar esta variable con detenimiento.

Finalmente, entre las limitaciones de esta revisión se encuentran en el uso de las bases de datos analizadas ya que pueden existir publicaciones indexadas en otras bases de datos, de igual forma en otros idiomas distintos al español e inglés, por lo que pudieron ser discriminados en este análisis. Además, al existir el sesgo de publicación, algunos trabajos científicos de calidad no se publican, se publican de forma tardía, presentan duplicidad en su publicación o cuentan con menos citas que otras publicaciones, estos son criterios que condicionan la búsqueda bibliográfica y afectar los resultados.

\section{CONCLUSIONES}

Los resultados de esta revisión sistemática aportan evidencia entre la relación de las características personales de los migrantes como posibles factores de riesgo que influyen en el consumo de drogas licitas e ilícitas. El uso de la metodología sistemática, la búsqueda estructurada y sistemática, basada en el análisis exhaustivo de la literatura disponible ayuda a la construcción de la evidencia que fortalecerá la aplicación y desarrollo de programas preventivos para este grupo específico, esta referencia es producto de la rigidez de la búsqueda de los criterios que permite clasificar e indagar en otras evidencias de la literatura, sobre todo al ser producción científica de alta calidad. Además, los hallazgos encontrados en esta revisión sistemática podrán ser una fuente de información para el desarrollo de nuevos estudios en población migrante.

Los autores de esta revisión recomiendan realizar otra revisión sistemática con el objetivo de identificar factores protectores que estén vinculados al consumo de drogas licitas e ilícitas ampliando las bases de datos y sin restricción del idioma, esto con el fin de generar evidencia literaria sintetizada que establezca aspectos orientados a la promoción de la salud y prevención del consumo de drogas. De igual forma, continuar indagando en factores asociados al consumo de drogas ilícitas en la población en estudio y discernir los estudios entre los grupos de migrantes de primera, segunda o tercera generación. 


\section{REFERENCIAS}

Arcury, T. A., Talton, J. W., Summers, P., Chen, H., Laurienti, P. J., \& Quandt, S. A. (2016). Alcohol Consumption and Risk for Dependence Among Male Latino Migrant Farmworkers Compared to Latino Nonfarmworkers in North Carolina. Alcoholism: Clinical and Experimental Research, 40(2), 377-384. https://doi.org/10.1111/acer.12969

Becoña Iglesias, E., España, Delegación del Gobierno para el Plan Nacional sobre Drogas, España, \& Ministerio del Interior. (2002). Bases científicas de la prevención de las drogodependencias. Ministerio del Interior. Delegación del Gobierno para el Plan Nacional sobre Drogas.

Blackson, T. C., De La Rosa, M., Sánchez, M., \& Li, T. (2015). Latino Immigrants' Biological Parents' Histories of Substance Use Problems in Their Country of Origin Predict Their Pre- and Post-Immigration Alcohol Use Problems. Substance Abuse, 36(3), 257-263. https://doi.org/10.1080/08897077.2014.932886

Bryant, A.N., \& Kim, G. (2013). The relation between acculturation and alcohol consumption patterns among older Asian and Hispanic immigrants. Aging \& Mental Health, 17(2), 147-156. https://doi.org/10.1080/13607863.2012. 727382

Calafat, A., Fernández Gómez, C., Juan, M., \& Becoña, E. (2005). Gestión de la vida recreativa: ¿Un factor de riesgo determinante en el uso reciente de drogas? Adicciones, 17(4), 337. https://doi.org/10.20882/adicciones.364

Cano, M. (2020). English use/proficiency, ethnic discrimination, and alcohol use disorder in Hispanic immigrants. Social psychiatry and psychiatric epidemiology. https://doi.org/10.1007/s00127-020-01837-5

Cano, M. Á., Sánchez, M., Rojas, P., Ramírez-Ortiz, D., Polo, K. L., Romano, E., \& De La Rosa, M. (2018). Alcohol Use Severity Among Adult Hispanic Immigrants: Examining the Roles of Family Cohesion, Social Support, and Gender. Substance Use \& Misuse, 53(4), 668-676. https://doi.org/10.1080/10826084.2017.1356333

Cantrell, J. (2014). A Multilevel Analysis of Gender, Latino Immigrant Enclaves, and Tobacco Use Behavior. Journal of Urban Health, 91(5), 928-939. https://doi.org/10.1007/s11524-014-9881-8

Chen, H.-H., \& Chien, L.-Y. (2018). Ethnic Drinking Culture, Acculturation, and Enculturation in Relation to Alcohol Drinking Behavior Among Marriage-Based Male Immigrants in Taiwan. American journal of men's health, 12(5), 1517-1529. https://doi.org/10.1177/1557988318772744

Creemers, H. E., Spanakis, P., Delforterie, M. J., \& Huizink, A. C. (2017). Alcohol use of immigrant youths in The Netherlands: The roles of parents and peers across different ethnic backgrounds. Drug and alcohol review, 36(6), 761-768. https://doi.org/10.1111/dar.12555

De La Rosa, M., Sanchez, M., Wang, W., Angel Cano, M., \& Rojas, P. (2020). Alcohol use trajectories of adult Latinx immigrants during their first decade in the United States. Addictive behaviors, 106, 106352. https://doi.org/10.1016/j. addbeh.2020.106352

Di Napoli, A., Morgillo, T., Rossi, A., Ventura, M., Nosotti, L., Cavani, A., Costanzo, G., Mirisola, C., \& Petrelli, A. (2020). Sociodemographic Characteristics Associated with Harmful Use of Alcohol Among Economically and Socially Disadvantaged Immigrant Patients in Italy. Journal of Immigrant \& Minority Health, 22(2), 426-431. https://doi. org/10.1007/s10903-019-00928-z

Dillon, F. R., De La Rosa, M., Sastre, F., \& Ibañez, G. (2013). Alcohol misuse among recent Latino immigrants: The protective role of preimmigration familismo. Psychology of Addictive Behaviors: Journal of the Society of Psychologists 
Cruz Gonzalez, A.A., Alonso Castillo, M.M., Armendáriz García, N.A. y Guzmán Facundo, F.R.

in Addictive Behaviors, 27(4), 956-965. https://doi.org/10.1037/a0031091

Hamilton, H. A., Danielson, A. M., Mann, R. E., \& Paglia-Boak, A. (2012). The Roles of Family, Peer, School, and Attitudinal Factors in Cannabis Use Across Immigrant Generations of Youth. Journal of Drug Issues, 42(1), 46-58. https:// doi.org/10.1177/0022042612436652

Harris, S., Dykxhoorn, J., Hollander, A.-C., Dalman, C., \& Kirkbride, J. B. (2019). Substance use disorders in refugee and migrant groups in Sweden: A nationwide cohort study of 1.2 million people. Public Library of Science Medicine, 16(11), e1002944. https://doi.org/10.1371/journal.pmed.1002944

Hernández, M. T., Sánchez, M. A., Ayala, L., Magis-Rodríguez, C., Ruiz, J. D., Samuel, M. C., Aoki, B. K., Garza, A. H., \& Lemp, G. F. (2009). Methamphetamine and cocaine use among Mexican migrants in California: The California-Mexico Epidemiological Surveillance Pilot. AIDS Education and Prevention: Official Publication of the International Society for AIDS Education, 21(5 Suppl), 34-44. https://doi.org/10.1521/aeap.2009.21.5_supp.34

Instituto Nacional de Psiquiatría Ramón de la Fuente Muñiz; Instituto Nacional de Salud Pública; Comisión Nacional Contra las Adicciones, \& Secretaría de Salud. Encuesta Nacional de Consumo de Drogas, Alcohol y Tabaco 20162017: Reporte de Alcohol. Villatoro-Velázquez, J.A., Resendiz, E. E., Mujica, S. A., Bretón-Cirett, M., Cañas-Martínez, V., Soto-Hernández, I., Fregoso-Ito, D., Fleiz-Bautista, C., Medina-Mora, M. E., Gutiérrez-Reyes, J., Franco-Núñez, A., Romero-Martínez, M. \& Mendoza-Alvarado, L. Ciudad de México, México, 2017. Recuperado de: https://www. gob.mx/salud\%7Cconadic/acciones-y-programas/encuesta-nacional-de-consumo-de-drogas-alcohol-y-tabaco-encodat-2016-2017-136758

Kaya, A., Iwamoto, D. K., Grivel, M., Clinton, L. \& Brady, J. (2016). The role of feminine and masculine norms in college women's alcohol use. Psychology of Men \& Masculinity, 17, 206-214. https://doi.org/10.1037/men0000017

Levitt, E., Ainuz, B., Pourmoussa, A., Acuna, J., De La Rosa, M., Zevallos, J., Wang, W., Rodriguez, P., Castro, G., \& Sanchez, M. (2019). Pre- and Post-Immigration Correlates of Alcohol Misuse among Young Adult Recent Latino Immigrants: An Ecodevelopmental Approach. International Journal of Environmental Research and Public Health, 16(22). https://doi.org/10.3390/ijerph16224391

Liberati, A., Altman, D. G., Tetzlaff, J., Mulrow, C., Gotzsche, P. C., Ioannidis, J. P. A., Clarke, M., Devereaux, P. J., Kleijnen, J., \& Moher, D. (2009). The PRISMA statement for reporting systematic reviews and meta-analyses of studies that evaluate healthcare interventions: Explanation and elaboration. British Medical Journal, 339(jul21 1), b2700b2700. https://doi.org/10.1136/bmj.b2700

Liu, Y., Song, H., Wang, T., Wang, T., Yang, H., Gong, J., Shen, Y., Dai, W., Zhou, J., Zhu, S., \& Pan, Z. (2015). Determinants of tobacco smoking among rural-to-urban migrant workers: A cross-sectional survey in Shanghai. BMC Public Health, 15(1), 131. https://doi.org/10.1186/s12889-015-1361-x

Liu, Y.-C., Chen, H.-H., Lee, J.-F., Chu, K.-H., \& Chien, L.-Y. (2017). Factors Associated With Drinking Behavior Among Immigrant Women in Taiwan. Substance Use \& Misuse, 52(5), 674-682. https://doi.org/10.1080/10826084.2016.1 253745

López-Tamayo, R., DiGangi, J., Segovia, G., León, G., Álvarez, J., \& Jason, L. A. (2016). Psychosocial Factors Associated with Substance Abuse and Anxiety on Immigrant and U.S. Born Latinos. Journal of addiction \& prevention, 4(1), 10.13188/2330-2178.1000028. https://doi.org/10.13188/2330-2178.1000028

Marsiglia, FlavioF., Kulis, S., Parsai, M., Villar, P., \& García, C. (2009). Cohesion and Conflict: Family Influences on Adolescent Alcohol Use in Immigrant Latino Families. Journal of Ethnicity in Substance Abuse, 8(4), 400-412. https:// doi.org/10.1080/15332640903327526

Health and Addictions / Salud y Drogas. Vol. 22 (1) 95-107 2022. 
Mulrow C.D. (1994). Rationale for systematic reviews. British Medical Journal. 309, 597-599. https://doi.org/10.1136/ bmj.309.6954.597

Muñoz-García, A., \& Arellanez-Hernández, J. (2015). Estrés psicosocial, estrategias de afrontamiento y consumo de drogas en adolescentes. Revista de Psicología y Ciencias del Comportamiento de la U. A. C. J. S, 6(2), 1-20. http:// www.scielo.org.mx/scielo.php?script=sci arttext\&pid=\$2007-18332015000200002\&lng=es\&tlng=es.

Organización Mundial de la Salud. Alcohol, Datos y Cifras: OMS; 2018. Recuperado de: http://www.who.int/es/ news-room/fact-sheets/detail/alcohol

Park, S.-Y., Anastas, J., Shibusawa, T., \& Nguyen, D. (2014). The Impact of Acculturation and Acculturative Stress on Alcohol Use Across Asian Immigrant Subgroups. Substance Use \& Misuse, 49(8), 922-931. https://doi.org/10.3109 /10826084.2013.855232

Patel, M., Mistry, R., Maxwell, A. E., Divan, H. A., \& McCarthy, W. J. (2018). Contextual Factors Related to Conventional and Traditional Tobacco Use Among California Asian Indian Immigrants. Journal of Community Health, 43(2), 280-290. https://doi.org/10.1007/s10900-017-0419-3

Patró-Hernández, R. M., Nieto Robles, Y., \& Limiñana-Gras, R. M. (2019). Relación entre las normas de género y el consumo de alcohol: Una revisión sistemática. Adicciones, 32(2), 145. https://doi.org/10.20882/adicciones.1195

Piñones-Rivera, C., Concha, N., \& Gómez, S. (2021). Perspectivas teóricas sobre salud y migración: determinantes sociales, transnacionalismo y vulnerabilidad estructural. Saúde e Sociedade. 30(1). Recuperado de: https://doi. org/10.1590/S0104-12902021200310

Sánchez, J. (2015). Alcohol use among Latino migrant workers in South Florida. Drug and Alcohol Dependence, 151, 241-249. https://doi.org/10.1016/j.drugalcdep.2015.03.025

Sánchez-Huesca, R., \& Arellanez-Hernández, J. L. (2011). Uso de drogas en migrantes mexicanos captados en ciudades de la frontera noroccidental México-Estados Unidos. Estudios fronterizos, 12(23), 9-26. Recuperado de: http:// www.scielo.org.mx/scielo.php?script=sci_arttext\&pid=S0187-69612011000100001\&lng=es\&tlng=es.

Sordo, L., Iciar Indave, B., Pulido, J., Molist, G., Rosales- Statkus, M. E., Ruíz-García, M., \& Barrio, G. (2015). Epidemiology of Alcohol Abuse Among Spanish Immigrant Populations. Revista Adicciones, 27(2), 132-140. http://dx.doi. org/10.20882/adicciones.697

Tortajada Navarro, S., Valderrama Zurián, J. C., Castellano Gómez, M., Llorens Aleixandre, N., Agulló Calatayud, V., Herzog, B., \& Aleixandre Benavent, R. (2008). Consumo de drogas y su percepción por parte de latinoamericanos. Psicothema [Internet], 20(3), 403-407. Recuperado de: http://www.psicothema.com/pdf/3499.pdf

Vázquez, F., \& Becoña, E. (2000). Factores de riesgo y escalada cannabinoide. Adicciones, 12(5), 175. https://doi. org/10.20882/adicciones.680

Walsh, S. D., Kolobov, T., \& Harel-Fisch, Y. (2018). Social Capital as a Moderator of the Relationship Between Perceived Discrimination and Alcohol and Cannabis Use Among Immigrant and Non-immigrant Adolescents in Israel. Frontiers in Psychology, 9, 1556. https://doi.org/10.3389/fpsyg.2018.01556

Wickramage, K., Vearey, J., Zwi, A. B., Robinson, C., \& Knipper, M. (2018). Migration and health: A global public health research priority. BMC Public Health, 18(1), 987. https://doi.org/10.1186/s12889-018-5932-5 
Cruz Gonzalez, A.A., Alonso Castillo, M.M., Armendáriz García, N.A. y Guzmán Facundo, F.R.

World Drug Report. (2020). Socioeconomic Characteristics and Drug Use Disorders (Booklet 5). United Nations Publication, Sales No. E.20.XI.6. Recuperado de: https://wdr.unodc.org/wdr2020/field/WDR20_BOOKLET_1.pdf

Zaller, N., Huang, W., He, H., Dong, Y., Song, D., Zhang, H., \& Operario, D. (2014). Risky alcohol use among migrant women in entertainment venues in China. Alcohol and Alcoholism (Oxford, Oxfordshire), 49(3), 321-326. https:// doi.org/10.1093/alcalc/agt184 